Horizons philosophiques

\title{
Genèse de la confession : la découverte de la conscience de soi comme mauvaise conscience
}

\section{Gaëlle Jeanmart}

Volume 10, numéro 1, automne 1999

Écritures et confessions

URI : https://id.erudit.org/iderudit/801108ar

DOI : https://doi.org/10.7202/801108ar

Aller au sommaire du numéro

Éditeur(s)

Collège Édouard-Montpetit

ISSN

1181-9227 (imprimé)

1920-2954 (numérique)

Découvrir la revue

Citer cet article

Jeanmart, G. (1999). Genèse de la confession : la découverte de la conscience de soi comme mauvaise conscience. Horizons philosophiques, 10(1), 59-84.

https://doi.org/10.7202/801108ar d'utilisation que vous pouvez consulter en ligne.

https://apropos.erudit.org/fr/usagers/politique-dutilisation/ 


\section{GENĖSE DE LA CONFESSION : LA DÉCOUVERTE DE LA CONSCIENCE DE SOI COMME MAUVAISE CONSCIENCE}

Cette étude est l'essai d'une phénoménologie de la confession qui tente d'en déterminer les caractéristiques propres. La confession n'est effectivement pas n'importe quel type de discours, c'est tantôt un acte littéraire comme chez Augustin et Rousseau, tantôt un acte de langage dans le secret de l'église ou du cabinet médical, comme dans la pratique confessionnelle chrétienne institutionnalisée au XVIIe siècle ou comme dans la psychanalyse freudienne; c'est, en tout cas, un récit en première personne et qui induit un rapport à soi tout particulier, fait à la fois de honte, de culpabilité et d'une humilité extrême qui donne le sentiment d'une certaine grandeur d'âme. Type particulier de rapport à soi, la confession est aussi rapport particulier à l'autre : le confesseur. Elle n'est jamais faite uniquement dans la plus pure intimité : la présence d'un regard extérieur celui du Dieu qui voit tout ou celui de la loi morale $-y$ est toujours sousjacente. Le dire de soi est un dire qui s'adresse toujours à un autre, lequel crée en retour un nouveau rapport à soi. La médiation de la confession de ses fautes par un interlocuteur au moins fictif instaure de moi à moi un rapport de distance. D'une distance qu'il faut d'ailleurs qualifier des termes de jugement, d'interdit, de condamnation ou de pardon. Ce rapport neuf qu'elle instaure de moi à moi et de moi à l'autre, j'ai voulu en trouver la genèse historique à travers l'émergence d'une problématique chrétienne, celle de la volonté libre et de l'autonomie que l'homme conquiert par rapport à Dieu. Cette volonté libre et le combat qui faisait rage en elle ont permis à l'homme de se constituer comme entité indépendante de Dieu mais dont l'indépendance n'était jamais que celle d'une tendance à la désobéissance qui faisait de lui un être hors-la-loi-divine. L'autonomie de la volonté, la découverte de la conscience de soi a donc été corrélative de la conscience de l'écart de I'homme par rapport à Dieu. La confession trace cette naissance ambiguë de la libre subjectivité comme liberté du mal; elle exhibe la convergence entre l'autobiographie et l'aveu des fautes, entre la conscience de soi et la mauvaise conscience. 


\section{De la raison à la volonté}

Dans la pensée grecque, jusqu'à Platon au moins, l'Univers est traversé par un logos créateur et ordonnateur. Même le démiurge de Platon ou le dieu unique de Xénophane restent soumis aux lois de ce logos et ne peuvent créer qu'un "kosmos", un univers harmonieux, et non chaotique. L'homme, créature raisonnable, participe au logos et peut donc comprendre sa propre position dans l'Univers, agir en fonction d'elle. Nul ne lui est besoin de connaître l'esprit ou la volonté du créateur. Sa raison lui suffit à appréhender dieu et le monde. Cette activité libre de l'intellect garantit la liberté morale. Elle conduit l'homme à l'intuition des lois immuables de l'ordre de l'Univers. Elle est la condition de l'ordre moral : aucune action ne peut être moralement mauvaise qui résulte d'une connaissance, et aucune ne peut être justifiée rationnellement qui soit mauvaise. II n'y a donc pas de modèle de vertu en Grèce qui soit forgé indépendamment du modèle épistémologique. ${ }^{1}$ Pour les tragiques, l'erreur morale est une aberration fatale $(\alpha \tau \eta)$, le mal, l'insolence (ußpis) et le châtiment, une loi naturelle et nécessaire $(v \varepsilon \mu \varepsilon \sigma \zeta))^{2}$ Pour Platon, le méchant est un malade et le péché, une erreur. ${ }^{3}$ La faute est une sorte de consentement arraché à l'âme par ses passions qui tiennent à son incarnation. Si, dans le Timée (81e-87a), il admet que la volition ( $\beta 0 v \lambda \eta, \beta 0 v \lambda \varepsilon \sigma \theta \alpha \mathrm{l})$ appartient à la partie immatérielle

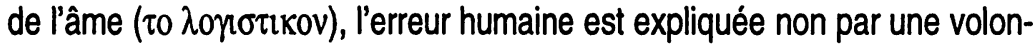
té mauvaise mais par une absence de connaissance due à la domination des parties matérielles et irrationnelles de l'âme sur la volonté intellectuelle. La volonté, soumise à l'intellect, est détruite par la sensibilité.

Or, si le péché est un péché d'ignorance, le sage peut-il pécher? Non, dans la mesure où il sait tout. ${ }^{4}$ Mais peut-il tout savoir? Les philosophes - à

1. Ou pour reprendre la formule célèbre de Socrate : «aucun homme ne peut faire le mal en connaissance de cause -". Les Grecs tendaient à faire du péché un acte inévitable, analogue aux accidents de la nature; le "fautif' pour eux n'était jamais loin du "fatal" : Effectivement "... la mentalité mythique (...) faisait de la chute et de la conversion des événements cosmiques sur lesquels la volonté personnelle ne pouvait rien" in Jean Guitton, Le temps et l'éternité chez Plotin et saint Augustin, p. 274.

2. Sur le sens de ces diverses notions et leur développement, on lira avec grand profit l'ouvrage de L. Gernet, Recherches sur le développement de la Pensée juridique et morale en Grèce, 1917, en particulier l'étude sémantique du terme nemesis et de son évolution, p. 305-347 (voir surtout 309, 314, 317, 323).

3. Protagoras, $357 \mathrm{c}-\mathrm{e}$, Lois, IX, $860 \mathrm{~d}$.

4. cf. également chez Plotin, chez qui l'idée est expressément exprimée dans les Ennéades, I, 8, §14, 1.23-I, 1, §9. 
commencer par Socrate - vont prendre conscience de limpossibilité pour l'homme de tout connaître, même - et surtout - pour le sage. Ils vont devoir réadapter leur conception de l'acte moral : il ne sera plus seulement déterminé par la pleine conscience de sa propre connaissance mais aussi par la pleine conscience de son ignorance. L'Apologie de Socrate (29 a) est symptomatique à cet égard : la peur de la mort vient de la supposition que la mort est un mal. Socrate propose de suspendre cette supposition puisqu'en réalité, on ne peut rien savoir sur la mort. Connaître sa propre ignorance de la mort permet de s'y préparer adéquatement. Stoïciens et épicuriens tireront des leçons de cette attitude. L'attitude grecque face au divin prend des accents pré-kantiens, stigmatisés dans la formule de Sénèque : "Non pareo deo sed assentior, je n'obéis pas au Dieu, je suis d'accord avec lui5". La position de l'homme vis-à-vis de Dieu n'est pas un acte de volonté - l'obéissance - mais le résultat d'un acte intellectuel reconnaissant l'ordre divin dans la nature. Cette allégeance à l'ordre divin n'est pas contraignante, ce n'est pas une soumission mais un acte libre de la raison. "Deo parere libertas est, le commerce avec Dieu est liberté6". On conçoit qu'il faille alors que l'ordre de la nature - ou la volonté de Dieu soient purement rationnels, et donc prévisibles.

En ce sens, la cosmologie biblique est complètement différente. Pour les chrétiens, la création résulte tout entière du pouvoir et de la volonté de Dieu. À la volonté de Dieu ne répond plus, en conséquence, l'intelligence de l'homme mais son obéissance ou sa désobéissance, sa foi ou son absence de foi. Lorsque Yahvé ordonne à Abraham de sacrifier son fils, Abraham ne réfléchit pas sur le sens de la demande qui lui faite, sens qui lui est d'ailleurs complètement occulté, il obéit; et la valeur de son obéissance tient, précisément, dans l'absence de toute justification rationnelle au commandement divin. Si agir en concordance avec sa raison continue de valoir pour la vie humaine, les standards de la vie morale sont édictés dans les commandements par lesquels Dieu a communiqué sa volonté aux hommes. Chez les Grecs, la foi était toujours évaluée bien en dessous de la connaissance (il ne faut pas croire en Dieu mais selon le bon mot de Sénèque être d'accord avec lui, c'est-à-dire connaître l'ordre des choses); chez les Chrétiens, la foi devient au contraire le point de référence pour déterminer la valeur morale d'une intention. Agir seulement en considération des fins et des moyens selon l'ordre rationnel du monde devient une

5. Sénèque, Ep., 96,2.

6. Sénèque, De vita beata, 15,3 . 
déficience dès lors que l'on pense que seul le pouvoir divin dirige l'ordre des choses et dispose le futur. Or, si les hommes ont cessé de croire fort orgueilleusement qu'ils pouvaient comprendre par leur propre intelligence les plans de Dieu, et ainsi l'ordre du monde, la raison a cessé d'être le facteur décisif de la vie morale. La volonté a détrôné la raison. ${ }^{7}$ On ne peut pas comprendre les commandements divins mais seulement leur obéir. Cette obéissance - ou cette désobéissance - provient d'une autre faculté que la faculté intellectuelle : la volonté libre, la volonté pour le bien comme pour le mal, la volonté de dire oui comme celle de dire non et de désobéir au commandement divin. Cette volonté libre pour le mal est capitale, elle suggère que la croyance et la foi présupposent toujours «la possibilité de la mécréance et de l'absence de foi ${ }^{8}$. En Grèce, on ne croyait pas réellement,

7. La volonté est le concept clé de la naissance de la conscience intime de soi. Chez les Grecs, les dieux sont à l'origine de toute action humaine. Ils débattent entre eux dans l'Olympe de ce qu'il convient de décider et puis descendent sur terre sous quelque forme et transmettent leur volonté aux hommes. L'action humaine n'a pas de commencement réel et autonome. On ne trouve pas chez Homère la conscience de la spontanéité de l'esprit humain, c'est-à-dire la conscience que les décisions relèvent de la volonté ou que les émotions et les sentiments ont leur origine dans l'homme lui-même. Goethe a souligné cette fonction du divin chez les Grecs, et notamment dans son entretien avec Riemer : "Ce que l'homme vénère comme divin n'est jamais que la projection à l'extérieur de ce qu'il y a de plus intime." II est par ailleurs bien connu que le grec n'a pas de mot pour désigner la volonté : "Le mot "volonté" (Wille) et ses équivalents dans les langues modernes ne trouvent aucun correspondant grec quand on les applique à la description et à l'évaluation de l'agir humain, l'impulsion à l'action (Handlungimpuls) sans considérer son origine possible dans une réflexion rationnelle ou une situation émotionnelle... La langue grecque ne dispose d'aucun terme pour désigner le phénomène de la volonté dans cet isolement (Isolierung)." (Albrecht Dihle, Die Vorstellung vom Willen in der Antike, Göttingen, 1985, p. 33). Ce livre brillant de M.A. Dihle entreprend de chercher les succédanés au terme de volonté et de montrer en quelle mesure ils ne pouvaient suffire à englober ce que le latin entend sous la terminologie "voluntas" et ensuite de montrer comment cette notion n'a pris son importance que dans un cadre de pensée chrétien et n'a vraiment fait son entrée dans le monde classique qu'avec saint Augustin. La thèse de Arendt - qui s'appuie sur la traduction anglaise de B. Snell, Die Entdeckung des Geistes, Hambourg, 1946, p. 155 - va volontiers en ce sens puisque dans la Vie de l'esprit elle fait d'Augustin le premier philosophe de la volonté (La Vie de l'Esprit, t. II, p. 48-53). Parmi les commentateurs moins illustres, R.-A Gauthier et J.-Y Jolif, Aristote. Ethique à Nicomaque, t. I, Louvain, 1959, p. 189-196, 211-212, partagent cette même opinion.

8. Bruno Snell, La découverte de l'esprit. La genèse de la pensée européenne chez les Grecs, trad. M. Charrière et P. Escaig, Paris : L'éclat (Polémos), p. 44. Et Snell de poursuivre : "La foi du credo implique l'existence d'une foi non orthodoxe, hérétique, dont elle se démarque. La foi est donc liée à un dogme pour ou contre lequel on combat. C'est quelque chose qui n'a pas existé en Grèce. Pour l'homme grec, les dieux sont si naturels et si évidents qu'il ne vien- 
on percevait comme évident, on savait. Le credo quia absurdum («je crois parce que c'est absurde" : autrement dit il n'y a de croyance que dans les actes de foi, donc précisément dans les cas litigieux où rien ne peut être prouvé) attribué à Tertullien ne pouvait s'écrire qu'en latin; il représente une polémique ouverte par les chrétiens contre la mentalité païenne des Grecs qui en venaient à supposer l'existence évidente des dieux à partir de l'ordonnance naturelle du monde. Et puisque l'ordre naturel s'imposait à la raison, il n'y avait pas à remettre en cause l'existence des dieux, ils s'imposaient comme s'imposent pour nous les vérités mathématiques. C'est vraiment le propre de la religion chrétienne, religion non-officielle née en marge des cultes officiels, de se fonder sur la croyance vécue comme acte de volonté, et donc comme acte de conscience. L'existence de Dieu ne s'impose plus naturellement, elle est l'acte de l'esprit qui refuse la naturalité et suppose l'existence du non-naturel, de l'inexplicable, du non-connaissable. Et cette absence de fond solide où l'on puisse cultiver ses certitudes contribue certainement à produire davantage de conscience de soi. $\mathrm{Ce}$ n'est plus dans l'ordonnance naturelle que l'homme va chercher les règles de sa conduite mais en lui-même. C'est à travers sa conscience de soi que l'homme peut devenir "conscient" de ce que Dieu veut de lui, c'est-à-dire : c'est à travers un acte de sa propre volonté que l'homme peut se soumettre à la volonté divine. La conscience de soi, c'est la présence de la volonté de Dieu dans l'individu. La conscience de soi est toujours une conscience morale. $^{9}$

Ainsi, chez les Grecs, c'est à travers un processus de connaissance que l'on connaît les dieux, on ne doit donc pas "croire" à leur existence, celle-ci est de l'ordre du connaissable. La faculté d'appréhension des dieux est chez eux une faculté rationnelle alors que chez les chrétiens elle tient à une foi non nécessairement - ou même, chez Tertullien, nécessairement non - fondée en raison. Chez les Grecs, parce que l'on connaît Dieu, on comprend rationnellement le sens de l'acte à poser, qui est simple mimétisme de la raison à l'œuvre dans tout projet, fût-il même divin; chez les chrétiens, parce que l'on croit en Dieu, on obéit à la volonté divine. À la

dra à l'idée de personne que d'autres peuples puissent avoir une autre foi ou d'autres dieux".

9. Ceci est valable pour le concept chrétien de conscience, il pourrait être intéressant de déterminer en quelle mesure cela reste vrai dans la suite de la tradition philosophique, et notamment chez Descartes et chez Locke en s'appuyant sur le beau commentaire d'E. Balibar à John Locke, Identité et Différence. L'invention de la conscience, Paris : Seuil, 1998. 
symétrie de l'homme grec qui imite son Dieu parce qu'ils ont en commun un logos rationnel répond la dissymétrie chrétienne entre le dieu qui ordonne et l'homme qui obéit aux commandements divins. Chez les Grecs, la moralité est aussi affaire de raison : on fait le mal par ignorance; chez les chrétiens, elle est affaire d'intention, on fait le mal parce que l'on veut le mal $^{10}$. À la connaissance grecque s'oppose enfin la volonté chrétienne ${ }^{11}$ :

$\begin{array}{ll}\text { Raison } & \text { Foi } \\ \text { Imitation } & \text { Obéissance } \\ \text { Connaissance } & \text { Volonté }\end{array}$

Et effectivement, tandis que chez les Grecs, la mauvaise action est toujours une action dans laquelle sont intervenus des éléments extérieurs à l'intellect humain qui l'ont submergé, et que l'action mauvaise est en conséquence une action dirigée par la fatalité et dont l'homme ne peut pas être tenu pour responsable, chez les chrétiens, l'intention qui préside à l'action est toujours libre, qu'elle soit bonne ou mauvaise, et la mauvaise action est donc à attribuer à la volonté libre de l'homme. En bref, chez les Grecs,

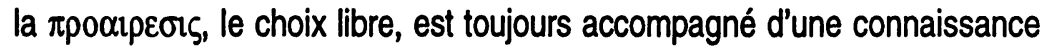
de la réalité qui montre une constante en toute chose. L'homme est capable de comprendre les règles éternelles du monde parce qu'il lui a été donné la même raison qui a servi de règle à la production par le dieu créateur de l'univers. Chez les chrétiens, le monde expérimenté par les hommes doit son existence à un créateur absolument libre. Et il est seulement dû à la bienveillance du créateur, et non à la rationalité à laquelle il serait soumis dans son exercice de création, que les processus cosmiques et naturels soient réguliers. Ainsi, l'homme ne doit pas d'abord expérimenter la rationalité et la régularité du monde, mais la volonté toute puissante du

10. On trouve illustrée cette différence dans un cas particulier du droit : chez les Grecs l'enquête sur la moralité d'un geste ou d'une parole porte uniquement sur le fait qu'ils ont été posés ou dits en connaissance de cause (la critique platonicienne de la sophistique n'est pas une critique de l'intention pervertie mais une critique de la doxa présentée sous l'apparence de savoir, d'epistèmè : si la sophistique est moralement déficiente c'est qu'elle instaure un mauvais critère de savoir - l'opinion humaine). Dans la juridiction romaine, en revanche, un argument portant sur l'intention et la volonté sera intégré à la technique du plaidoyer; et c'est désormais la volonté et l'intention plutôt que le fait et sa constatation qui deviennent le point de référence auquel la lettre des lois doit être appliquée.

11. Il est évident qu'ici encore le morale aristotélicienne ne peut être intégrée à ce schème rapide sans plus de précautions. Cf. notamment les critique de B. Cassin à l'encontre de l'analyse arendtienne de la proairesis, in Aristote et le logos. Contes de la phénoménologie ordinaire, Paris : P.U.F., 1997, p. 72 sq. 
créateur. Et cette expérience est elle-même un acte de volonté : les hommes ne peuvent pas connaître rationnellement tout ce que cache la volonté divine. Ils peuvent seulement donner une réponse aux commandements divins par l'obéissance ou la désobéissance c'est-à-dire par la volonté. Ainsi, la volonté de l'homme est née du commandement de Dieu. La souveraineté de Dieu qui est au-dessus de l'entendement humain demande de la part de l'homme un acte de volonté, c'est-à-dire une obéissance ou une désobéissance. À la volonté de Dieu, l'homme répond lui-même par un acte de volonté : l'obéissance et la foi.

\section{Saint Paul et le conflit des volontés}

Peut-être faut-il formuler cette phrase sous sa forme négative : sans la volonté de Dieu, pas de volonté humaine, ou pour reprendre la formulation paulinienne du problème :

\section{Où il n'y a pas loi il n'y a pas transgression ${ }^{12}$ (...) je n'ai connu le péché que par la Loi. Je n'aurais pas su la convoitise si la Loi n'avait dit : tu ne convoiteras pas. Mais le péché a pris occasion du comman- dement pour produire en moi toute convoitise, car sans la loi le péché est mort. Moi je vivais sans loi autrefois; mais quand le commande- ment est venu, le péché a pris vie. ${ }^{13}$}

Ce texte laconique repose sur toute une série de présupposés non élucidés. Que la loi ait créé le péché implique que son ordre n'était pas contraignant et qu'à son commandement elle présupposait donc la volonté libre de l'homme d'obéir ou non. Si à la loi, ma réponse a été la convoitise, c'est que cette loi du créateur laissait la place à la liberté de la créature. Elle n'était loi qu'à pouvoir être transgressée. Et c'est sur cet écart entre la loi divine et la réponse humaine à la loi que repose d'ailleurs la foi. La foi est rendue possible seulement par la volonté libre et donc par la possibilité de la désobéissance. Le "Tu Voudras" de la Loi, son impératif moral, implique mon choix : «je veux" ou "je ne veux pas" c'est-à-dire "j'obéis" ou «je n'obéis pas". Et la transgression, le "je ne veux pas" est toujours la réponse qui signe le plus évidemment ma volonté propre.

On pourrait dire, en suivant l'interprétation qu'en donne Arendt, que l'alternative qui se présente à la volonté libre de l'homme est plus complexe que l'alternative entre oui et non, entre "j'obéis" et "je désobéis". En réalité, l'alternative n'est pas tant «je veux ou je ne veux pas» que "je veux

12. Épitre aux Romains, IV, 15.

13. Epitre aux Romains, VII, 7-9. 
et je ne veux pas" : la réflexivité n'est jamais autant marquée que dans la volonté où s'entame toujours une lutte intérieure entre l'obéissance et la désobéissance, la libre soumission ou la rébellion mauvaise et nocive. "Chaque Je-veux se heurte inévitablement à un Je-non-veux, tant et si bien que même si on obéit à la loi et y satisfait, il subsiste une résistance intérieure ${ }^{14}$ ». Volonté et Réflexivité sont au plus étroitement liées chez saint Paul. Plus que par tout autre acte, l'homme se prend lui-même pour objet dans un acte de volonté. Non seulement parce que la volonté est la faculté de l'autonomie et de la liberté et qu'elle ne s'ordonne ainsi qu'à elle-même, à la différence de la faculté rationnelle qui dépend de l'ordre des choses; mais aussi parce que la volonté est le lieu d'un conflit intérieur qui rend l'homme conscient de lui-même par les tensions qui l'animent. Je suis seulement conscient de mon pied lorsqu'il me fait mal; je suis seulement conscient de moi à travers mon tourment et ma culpabilité, à travers le conflit intérieur qui me déchire. Parce que je sens en moi, intimement, le conflit des volontés, je me déprends du monde extérieur pour écouter mes doutes et mes hésitations. L'être humain est un être nécessairement déchiré, impuissant à gérer lui-même son conflit intérieur. Le problème ne peut jamais être résolu en obéissant à la loi ou en cédant au péché; selon Paul, ce conflit interne ne peut être raccommodé que par la Grâce, gratuitement. Demeurer volontairement "dans tout ce qui se trouve écrit au livre de la Loi, pour le pratiquer» est «impossible $\mathrm{e}^{15}$. . Oublier tout à fait la loi ou se consacrer pleinement au péché est également impossible au chrétien qui se tient toujours dans un entre-deux. Le doute porte sur tout acte humain. Et ce doute s'introduit d'autant plus profondément que c'est la volonté qui est en question dans le péché humain, et que, pour juger de la valeur morale de l'acte - c'est-à-dire de l'intention intime et cachée qui préside à l'acte et non de l'acte, lui-même manifeste - c'est cette volonté qu'il faudra observer. La volonté, c'est l'intimité, le cœur, le dedans de l'homme, et non l'apparence, ce qui est extérieur et visible. C'est dans cette vigilance dont il a désormais fallu entourer non seulement nos actes, mais aussi nos pensées intimes et nos intentions cachées, que la conscience de soi a trouvé son plus sûr ferment. Cette vigilance est née de la certitude que Dieu connaît l'intérieur de nos cœurs et n'observe pas simplement à l'extérieur ce qu'on laisse apparaître. Dieu juge nos intentions - qui, perverses, sont

14. Hannah Arendt, La Vie de l'Esprit, vol. II : La Volonté, Paris : P.U.F. (philosophie aujourd'hui), 1983, p. 87.

15. Épitre aux Galates, 3, 10. 
coupables - en plus de nos actes et de leur mauvais résultat. De sorte que l'on peut pécher sans prononcer une parole et sans esquisser le moindre geste ${ }^{16}$. Pour Jésus le péché peut être consommé dans le cœur sans avoir au dehors son effet ${ }^{17}$. Selon Saint Paul : "Les hommes montrent l'œuvre de la Loi inscrite dans leurs cœurs, comme en témoignent leur conscience et leurs pensées qui les accusent ou les disculpent jusqu'au jour où, selon mon évangile, Dieu jugera par Jésus Christ le dedans des hommes ${ }^{18}$ ".

Le monde des hommes est peut-être un monde de l'apparence où le rusé peut tromper mais la ruse n'abuse pas Dieu qui perce l'apparence et qui décèle l'intime sous le superficiel : "N'est pas juif celui qui en a l'apparence, n'est pas circoncision ce qui en a l'apparence dans la chair. Mais le Juif l'est au-dedans, la circoncision est du cœur, en esprit et non à la lettre : à lui, l'éloge vient non des hommes mais de Dieu ${ }^{19}$ ".

Non seulement l'intériorité est plus perceptible parce qu'elle est le lieu d'un conflit, mais aussi parce que l'issue de ce conflit fixe mon sort lors du jugement divin. J'ai plus conscience de moi à travers la tension insoluble de mes diverses volontés; et j'ai d'autant plus conscience de cette tension qu'y sont forcément intégrés les volontés de désobéissance, les mouvements récalcitrants à la Loi, et qu'intimement je sais que Dieu connaît cette résistance et qu'il la condamne.

\section{La confession d'Augustin}

Il était intéressant, m'a-t-il semblé, de formuler une hypothèse sur la naissance de la volonté dans le monde chrétien afin de voir quelle gangue l'entourait de manière constitutive. II ressort de cette hypothèse que la conscience de soi est nécessairement, par et depuis son acte de naissance, une conscience malheureuse. Et cela à deux titres et pour deux raisons : la conscience de soi est mauvaise conscience d'abord parce qu'elle est inéluctablement divisée, qu'elle est rongée par le doute et par la tension entre des pôles opposés. Elle n'est donc jamais une pure et simple

16. Et ici, je serais encore d'accord avec l'interprétation de Arendt lorsqu'elle conclut : "Ainsi, quand on aborde saint Paul, l'accent passe totalement du faire au croire, de l'homme extérieur, vivant dans un monde de phénomène (...) à une intériorité, qui, par définition, ne se manifeste jamais sans équivoque et ne peut-être passée au crible que par ... Dieu." Hannah Arendt, La Vie de l'Esprit, vol. II : La Volonté, Paris : P.U.F (philosophie aujourd'hui), 1983, p. 85.

17. Mt., V, 27-28; XII, 35; Luc, VI, 43-45.

18. Épitre aux Romains, II, 15-16. 
obéissance à la Loi divine, il y a toujours en elle au contraire l'attraction de l'interdit. La conscience de soi est mauvaise conscience parce qu'elle est inéluctablement attirée par la désobéissance et parce qu'elle est en outre et tout aussi inéluctablement - consciente de sa faute et du regard que Dieu porte sur celle-ci. La conscience de soi est donc rongée à la fois par le doute et par la culpabilité qui résulte de cette impossibilité à choisir la bonne voie, la foi divine, d'emblée et sans hésitation.

Toutes ces questions que saint Paul aborde sans sembler être conscient des implications philosophiques ou des développements rationnels qu'il convient d'apporter à son discours, Augustin les reprend avec beaucoup plus de maîtrise. Ce qui est intuition obscure chez Paul devient chez Augustin argument irréfutable. Mais on sent néanmoins le lourd héritage de saint Paul à travers la manière augustinienne d'aborder la problématique de la volonté : il s'agirait pour Augustin de reprendre saint Paul pour le porter à la philosophie. Ainsi par exemple la caractérisation paulinienne du conflit des volontés comme conflit entre chair et esprit est soigneusement reprise et méditée par Augustin dans ses Confessions. Chez saint Paul, le conflit des volontés était un conflit entre la volonté charnelle, concupiscente et la volonté spirituelle, qui est une volonté du bien. ${ }^{20}$ Augustin découvre, lui, que l'interprétation de saint Paul d'une lutte entre chair et esprit est fausse : "Mon corps obéissait plus aisément à la plus ténue volonté de mon âme, que mon âme ne s'obéissait elle-même pour réaliser dans la volonté seule sa grande volonté21». Et Augustin de s'interroger : "D'où vient ce prodige? Quelle en est la cause? (...) L'âme commande au corps et elle est directement obéie, l'âme se commande à

19. Épitre aux Romains, II, 28-29.

20. Si la faute est en moi, c'est par le corps : “18. Je sais en effet que le bien n'habite pas en moi, c'est-à-dire dans ma chair, car vouloir le bien est à ma portée, mais non l'accomplir. 19. Ainsi le bien que je veux je ne le fais pas, mais le mal que je ne veux pas, je le pratique. 20 . Si donc je fais ce que je ne veux pas, ce n'est plus moi qui agis mais le péché logé en moi. 21. Je trouve donc cette loi, pour moi qui veux faire le bien, que le mal est à ma portée; 22. Car en mon for intérieur je me délecte de la loi de Dieu; 23. mais je vois dans mes membres une autre loi mener la guerre contre la loi de mon intelligence et me faire prisonnier de la loi du péché qui est dans mes membres. 24. Misérable de moi ? qui me délivrera du corps de cette mort? 25. Dieu merci par Jésus Christ notre seigneur ! Ainsi l'intelligence m'asservit-elle à la loi de Dieu et la chair à la loi du péché." (Epitre aux Romains, chapitre VII). Ici viendrait s'installer un mépris du corps, déjà entériné par le pythagorisme, le platonisme et le stoīcisme, et qui est toujours sous-jacent chez les grands penseurs chrétiens, chez Anselme, Abélard ou Thomas.

21. Confessions, VIII, 8. 
elle-même et elle rencontre une résistance?» Peut-être, poursuit-il plus loin, est-ce que l'esprit «(...) ne commande pas pleinement : voilà pourquoi son ordre ne s'exécute pas (...) et c'est pourquoi ce qu'il commande n'est pas." Mais qui commande alors? L'esprit ou la volonté? Est-ce que l'esprit donne des ordres à la volonté, et se faisant hésite, si bien que la volonté ne reçoit pas d'ordre équivoque? La réponse est non, car : «la volonté appelle à l'être une volonté qui n'est pas autre chose qu'elle-même ${ }^{22}$ ".

La fissure s'instaure dans la volonté même; le conflit n'est dû ni à la rupture entre esprit et volonté, ni à celle entre chair et esprit. Le fait même que la volonté s'exprime toujours à l'impératif en témoigne : "Tu voudras" ordonne la volonté à elle-même. Elle seule a le pouvoir de donner de tels ordres, et "si l'âme se mettait tout entière dans son commandement, elle n'aurait pas besoin de se commander d'être". C'est dans la nature de la volonté de se dédoubler. Chaque fois qu'il y a volonté, «il y a deux volontés dont aucune n'est complète (tota), et ce qui manque à l'une, l'autre le possède". Disant "Tu dois" à ma volonté, je suscite en retour un autre "Tu dois" qui répond au premier. Ou plus exactement ma volonté se scinde nécessairement en deux, en une première volonté maîtresse qui exprime le "tu dois" et en une autre volonté, esclave de la première, qui obéit ou n'obéit pas à l'injonction 23 . II faut toujours deux volontés rivales pour vouloir : "cette volonté partagée qui veut à moitié et à moitié ne veut pas n'est donc nullement un prodige." L'ennui est que c'est le Moi voulant qui veut et non-veut simultanément : “C'était moi qui voulait et c'était moi qui ne voulait pas... Ni je ne disais pleinement oui, ni je ne disais pleinement non" - ce qui ne signifie pas que «j'avais deux âmes, ayant chacune leur nature, l'une bonne, l'autre mauvaise", mais que les assauts de deux volontés dans une seule et même âme "me déchirent24».

On voit bien combien saint Augustin est plus rigoureux que saint Paul; et cette rigueur on la retrouve aussi dans la généalogie de la volonté. Chez saint Paul, la volonté humaine naît en réaction à la loi qui s'énonce sous la forme contraignante du «Tu dois». Mais il n'est pas expliqué pourquoi l'homme dispose d'une marge de liberté lui permettant de prendre une distance réflexive avec cet ordre et d'envisager la possibilité de ne pas lui obéir. II ne va pas de soi qu'à l'ordre de Dieu, on ne soit pas forcé d'obéir 22. Confessions, VIII, 8.

23. Ou pour reprendre encore les termes de Arendt, La vie de l'esprit, p. 116 : “... puisqu'il est dans la nature de la volonté d'ordonner et d'exiger l'obeissance, c'est aussi dans sa nature d'être l'objet de ses résistances".

24. Confessions, livre VIII, chap. 9 et 10. 
tout de go sans même avoir le sentiment d'obéissance, mais comme sous le fait d'une contrainte naturelle inévitable. Si la loi suscite la résistance de l'homme, c'est que celui-ci est déjà doté d'un libre arbitre, qu'il a déjà au moins comme potentielle la possibilité de résister. Pas plus qu'ailleurs Paul n'affronte-t-il ici la question philosophique sous-jacente.

Cette question est pourtant déjà ancienne. Elle est née avec Platon dès que s'est envisagée la notion de perfection divine. Le monde matériel, le monde appréhendé par nos sens, est selon Platon de peu de consistance réelle, c'est un monde de changement loin d'être parfaitement organisé. Et tout s'organise chez Platon selon une structure pyramidale qui place au sommet l'être absolument réel, le monde des idées, et en décroissance de réalité tout ce qui est rongé par le devenir. Et cet être suprême au sommet de la pyramide, Platon le décrira dans la République comme extérieur au domaine des étants, de forme indéterminée, causé par - ou relié à - aucune autre chose. Ce sont indubitablement là les racines de l'idée d'être absolu et omnipotent. De cette perfection et de cette omnipotence divine, on peut formuler une problématique qui sera assumée seulement par la suite. Si Dieu est parfait et qu'il est omnipotent, pourquoi l'homme peut-il être libre pour le mal? S'il est tout puissant, Dieu est créateur de toute chose : il n'est rien qui ne soit sans sa libre volonté, mais alors, pourquoi un Dieu bon et juste permet-il l'injustice et le péché? Pourquoi y a-t-il de la souffrance et de la misère sur terre?

Les néoplatoniciens hériteront du problème; Plotin, notamment, qui le résoudra de la manière suivante : le vrai univers de l'esprit est totalement bon, mais dieu devait créer et ce qu'il créa devait forcément être moins bon que lui-même ${ }^{25}$. Le mal est donc le prix de la variété. Mais pourquoi alors y a-t-il tant de maux ? Augustin affrontera cette fois le problème : il est juste que certains souffrent; la souffrance est une juste punition du péché26. Mais si la souffrance est la conséquence du péché, pourquoi alors de très jeunes enfants peuvent-ils souffrir? Pourquoi cela serait-il juste alors que, précisément, ils n'ont probablement pas encore eu l'occasion ni le temps de pécher?

Lorsqu'on en vient à la peine des petits enfants, crois-moi, je ne vois pas comment en sortir et je ne peux vraiment pas trouver de réponse.

25. Ennéades, 3, 2, 278/279.

26. Cf. De Ord., II, 4, 12 ; Sol., 1, 1.2; Lib. Arb., III, 9, 26. 
(...) Ils sont affaiblis par la maladie, abattus par la peine, tourmentés par la faim et la soif, disloqués, empestés par des esprits malsains. Indubitablement, il doit être montré qu'ils ont enduré tout cela justement alors que la cause n'est pas de leurs mauvaises actions. II faut dire que ces choses arrivent sans que Dieu ne le sache, ou sans qu'il puisse enrayer leurs causes, ou qu'il les cause et les permet injustement. (...) On doit donc être capable de référer à une juste cause tous ces malheurs qui arrivent aux jeunes enfants. Bien sûr lorsque des personnes plus âgées sont affligées ainsi on peut dire soit que leurs mérites sont testés, comme c'est le cas de Job, soit que leurs péchés sont punis, comme c'est le cas de Hérode; et de ces cas particuliers où Dieu a voulu infliger une peine, on peut en inférer aux autres cas, qui sont plus obscurs. Mais ce sont de vieilles personnes. Dis-moi comment peut-on répondre à propos de jeunes enfants, s'il n'y a aucun péché à punir de toutes ces souffrances, et s'il ne faut manifestement pas encore tester leur tempérance à cet âge 27 " .

La réponse d'Augustin viendra plus tardivement. Et c'est finalement la seule réponse qu'il pouvait donner en conservant les bases théoriques qui sont ici formulées, c'est-à-dire en conservant la toute puissance et la parfaite bonté de Dieu : la seule alternative possible pour le chrétien s'il veut justifier Dieu, c'est de croire à sa propre culpabilité. Le syllogisme est incontournable :

(1) Tous les hommes souffrent

(2) Toute souffrance est une punition divine

(3) Tous les actes de Dieu sont justes

(4) Qui est puni sans avoir péché est puni injustement

CQFD : Tous les hommes commettent le péché.

Tous : y compris les jeunes enfants; c'est la raison pour laquelle les Confessions s'ouvrent sur cette affirmation : «Personne n'est devant toi pur de péché, pas même l'enfant qui n'a sur terre vécu qu'un seul jour ${ }^{28}$ ".

Pour que les hommes pèchent contre la volonté de Dieu, il faut qu'ils soient eux-mêmes dotés d'un libre arbitre, d'une volonté propre. Ainsi, la volonté des hommes, c'est une volonté pour le péché, la liberté humaine, une liberté pour le mal. L'autonomie du sujet n'est instaurée que pour expliquer le mal et la peine sur terre; c'est sans plus une autonomie du mal, une autonomie pour le mal. Et c'est là tout le paradoxe chrétien : être obligé de créer la subjectivité humaine autonome, la libre volonté de l'homme,

27. "Lettre à Jérôme», Epistolae, 166, 6, (je traduis).

28. Confessions, I, VII, 11; cf. jb, 14, 4 et sq (Septante). 
mais de les créer seulement pour déprendre Dieu de l'origine du péché. Se vouloir "Je", c'est se vouloir - et déjà être - pécheur, pour que Dieu ne puisse être tenu pour responsable de nos malheurs innocents. L'autosubjectivité est d'emblée marquée par la culpabilité; et le dire de soi est marqué de la même ambivalence : il est créé seulement par les chrétiens mais comme expiation, comme dire de la faute originelle et inéluctable. Le dire de «Je", l'autobiographie, est donc nécessairement le dire des péchés et des fautes : l'autobiographie devait nécessairement prendre chez Augustin la forme de la confession.

L'exercice de la confession part toujours d'une évidence : le péché. C'est ce péché qu'il faut confesser avec l'humilité de celui qui connaît son imperfection (la première occurrence du terme "confesser" dans les Confessions d'Augustin advient dans ce contexte : "mon âme était trop étroite, elle est en ruine, elle blesserait donc ton regard, je le sais, je le confesse»). II faut se confesser à Dieu, l'être parfait, seul capable de remettre et de pardonner le péché. II faut, dit Augustin «dénoncer devant lui (devant Dieu) mes délits, et cela contre mo29". La confession faite de l'être imparfait vers l'être parfait est une pratique humiliante, la pratique caractéristique peut-être d'une religion de martyrs. Parler contre soi-même, voilà bien qui définit l'aveu extorqué, et l'acte de contrition. Le dire de soi est punition.

Le premier philosophe à créer la subjectivité et à consacrer l'autonomie du sujet les crée aussi comme pure négativité. Dire "Je», c'est déjà nier Dieu, alors que paradoxalement je suis obligé de dire «je» et de consacrer mon autonomie pour assurer à Dieu sa perfection, puisque sans moi, sans mon libre arbitre, c'est Dieu qui est la cause du malheur de l'homme sans que celui-ci n'ait rien fait pour le mériter. Sans le «je» libre, c'est Dieu qui soit a voulu que je pèche, ce que j'ai donc fait involontairement, soit me punit injustement en m'assignant mon lot de souffrances imméritées puisque, n'ayant pas d'autonomie, je n'ai pas pu faire le mal volontairement. Cette obligation ouvre la possibilité à toute une phénoménologie des actes de conscience de naître (mais il faut bien voir que l'origine de cette phénoménologie est négative). On sort enfin de la manière transcendante d'expliquer le monde, on sort du don d'ubiquité attribué au philosophe. $\mathrm{Ce}$ ne sont plus de grands systèmes théoriques qui peuvent donner l'ordonnance du monde, systèmes qui reposent uniquement sur l'organisation a priori de la raison, c'est l'expérience que fait le sujet du monde. Désormais

29. Confessions, I, V, 5 (je souligne). 
on peut partir de sa propre perception parce que l'on pense que l'ordre du monde n'est plus à disponibilité, parfaitement adéquat à l'ordre de notre raison humaine. On peut partir du sujet parce que le sujet est doté d'une autonomie qui l'autorise à rattacher son savoir à lui-même et parce qu'il ne peut désormais plus espérer connaître l'Univers sans passer par l'appréhension de la volonté divine à travers sa propre volonté.

La culpabilité serait bien la condition de possibilité historique de cette phénoménologie augustinienne, et la confession sa première formulation. Foucault avançait dans son Histoire de la sexualité une opinion assez proche de la mienne mais qui concernait cette fois la pratique institutionnalisée de l'aveu au XVIIe siècle. Cette pratique confessionnelle, concluait Foucault, avait sans doute conduit à :

\begin{abstract}
une métamorphose dans la littérature : d'un plaisir de raconter et d'entendre, qui était centré sur des récits héroĩques ou merveilleux des "épreuves" de bravoure ou de sainteté, on est passé à une littérature ordonnée à la tâche infinie de faire lever du fond de soi-mème, entre les mots, une vérité que la forme mème de l'aveu fait miroiter comme inaccessible. De là aussi, cette autre manière de philosopher: chercher le rapport fondamental au vrai, non pas simplement en soi-même - dans quelque savoir oublié ou dans une certaine trace originaire - mais dans l'examen de soi-même qui délivre, à travers tant d'impressions fugitives, les certitudes fondamentales de la conscience. ${ }^{30}$
\end{abstract}

C'est évidemment à Descartes et ses Meditationes de prima philosophia que pensait Foucault. Mais comment ne pas penser aussi aux méditations de l'évêque d'Hippone, qui à défaut d'avoir institutionnalisé la pratique confessionnelle comme sacrement ecclésiastique i'avait au moins institué comme acte littéraire. C'est donc déjà avec Augustin, au IVe siècle, que naît la pratique de l'aveu si répandue au XVIIe siècle, sur laquelle Foucault s'est penché dans son livre sur L'histoire de la sexualité. C'est déjà à mon sens avec Augustin, avec ces premières "confessions", que s'impose sous la forme d'une contrainte religieuse cette injonction que Foucault dit être «si particulière à l'Occident moderne (...) la tâche, quasi infinie, de dire, de se dire soi-même et de dire à l'autre, aussi souvent que possible ${ }^{31}$ ». Avec la pratique de la confession, Augustin donne aussi naissance à la pratique de l'autobiographie, à un nouveau genre littéraire, à un nouveau type de philosophie.

30. Michel Foucault, Histoire de la sexualité, Paris : Gallimard, 1976, p.80.p. 80.

31. Michel Foucault, ibid., p. 29. 


\section{À Dieu et aux hommes}

Que la confession d'Augustin, comme celle de Rousseau d'ailleurs, soit un acte littéraire n'a rien d'anodin. II amène avec lui la préoccupation de l'interlocuteur de ces confessions. C'est à Dieu bien sûr que ces deux confessions s'adressent. II est à chaque fois l'interlocuteur majeur et le premier destinataire de l'œuvre écrite. Mais si cette œuvre est écrite, c'est pour les hommes et non pour Dieu qui eût pu se contenter d'une confession faite dans le silence du cœur : Je veux "faire la vérité" dans mon cœur, devant toi par la confession, mais aussi dans mon livre, devant de nombreux témoins. 32 De même, au début de ses confessions, Rousseau déclarait-il : "(...)j'ai dévoilé mon intérieur tel que tu l'as vu toi-même. Être éternel, rassemble autour de moi l'innombrable foule de mes semblables: qu'ils écoutent mes confessions, qu'ils gémissent de mes indignités, qu'ils rougissent de mes misères. Que chacun d'eux découvre à son tour son cœur aux pieds de ton trône avec la même sincérité33".

L'acte littéraire des confessions augustiniennes et rousseauistes font d'elles des discours publics, non des discours ou je parle de "je" dans l'intimité de la conscience intérieure. On n'a pas saisi le phénomène confessionnel dans son ensemble (on ne l'a pas saisi dans son sens plein d'acte littéraire et public) quand on l'a défini comme un rituel de discours à la première personne et qu'on l'a associé à la naissance de la conscience de soi comme mauvaise conscience. Le sujet qui parle coïncide avec le sujet de l'énoncé, c'est entendu; mais cette coïncidence entre le sujet et l'objet du discours pose problème. Pourquoi instaurer de moi à moi ce rapport de distance objectal, alors que dans cette distance qu'installe le discours autobiographique on ne voit plus très bien l'intérêt du discours? Pourquoi dire ce que déjà on n'est plus? Pourquoi dire dans une reconstruction artificielle qui lie une chaîne de gestes ou de pensées inchoatifs en une linéarité ce qui se présente toujours sous forme multiple, diffuse et infusée? Pourquoi ou plutôt pour qui ? Cette question de l'interlocuteur du discours confessionnel est capitale. L'aveu de confession n'est pas celui que l'on se fait à soi-même. La distance induite par l'aveu : je dis ce que l'autre veut, ou ne veut pas entendre, fait de lui un mode de discours résolument interpersonnel. L'aveu que je me fais à moi-même est toujours médié - fut-il d'ailleurs même silencieux - par un principe collectif, interdit moral ou loi

32. Augustin, Confessions, $\mathrm{X}, 1$.

33. Jean-Jacques Rousseau, Les Confessions, Paris : Gallimard, Bibliothèque de la Pléiade, p. 1. 
familiale. On ne confesse rien sans la présence au moins virtuelle d'un interlocuteur qui est l'instance qui requiert l'aveu, qui le jugera et qui pardonnera ou punira. La confession est éminemment un lieu de pouvoir. Le pouvoir de l'impératif moral qui s'impose dans l'intimité du sujet. "La loi morale en moi»" : voilà bien un principe-clé de la confession. Elle est toujours une parole obligée, une parole forcée, faisant sauter par quelque contrainte impérieuse les sceaux de la retenue ou de l'oubli.

Pour Augustin et pour Rousseau, l'impératif confessionnel est d'abord un impératif divin : il faut avouer ses errements pour être pardonné de Dieu : "(...) à qui d'autre que toi irais-je proclamer : de mes fautes cachées, purifie-moi, Seigneur (...) N'ai-je pas dénoncé devant toi mes délits, et cela contre moi, mon Dieu? Ne m'as-tu pas remis de l'impiété de mon cœur? ${ }^{34}$ ". La confession est adressée à Dieu parce qu'à l'aveu de ses péchés, le pécheur espère voir succéder immédiatement le pardon de Dieu. Chez Rousseau, le but de la confession écrite est celui-là aussi. L'introduction témoigne que la perspective est bien la perspective eschatologique chrétienne du jugement dernier : "Que la trompette du jugement dernier sonne quand elle voudra; je viendrai ce livre à la main me présenter devant le souverain juge. Je dirai hautement : voilà ce que j’ai fait, ce que j'ai pensé, ce que je fus. J'ai dit le bien et le mal avec la même franchise. Je n'ai rien tu de mauvais, rien ajouté de bon..."

Rien tu de mauvais, rien ajouté de bon : l'aveu est lucide, froid et ne tente jamais d'épargner son confesseur. Chez Rousseau aussi, il s'agit d'avouer contre soi-même à Dieu et aux hommes; et cela parce que l'on a contrevenu à une loi divine et humaine. Chez Augustin, il n'y a qu'un péché majeur, celui de n'avoir pas cru en Dieu, et d'avoir mené une grande part de son existence à l'écart de la loi divine. La confession signale donc d'ellemême la conversion : la fin de l'errance hors-la-loi-divine. Chez Rousseau, de même, c'est l'aveu lui-même, parce qu'il est public, qui procure l'apaisement de la conscience coupable. Ce que Rousseau considérait comme le plus noir péché de sa vie - le vol d'un petit ruban rose et argent et surtout l'incapacité d'avouer sa faute et la lâcheté d'en avoir chargé quelqu'un d'innocent pour échapper à la honte de l'aveu public ${ }^{35}$ - il n'avait jamais pu l'avouer à personne :

34. Augustin, Confessions, I, 5.

35. On voit bien ici que le châtiment - l'écriture même des Confessions - épouse le crime : la honte d'avouer. 
Je n'ai jamais pu prendre sur moi de décharger mon cœur de cet aveu dans le sein d'un ami. La plus étroite intimité ne me l'a jamais fait faire à personne, pas même à Madame de Warens. Tout ce que j'ai pu faire a été d'avancer que j'avais à me reprocher une action atroce, mais je n'ai jamais pu dire en quoi elle consistait. Ce poids est donc resté jusqu'à ce jour sans allégement, sur ma conscience, et je puis dire que le désir de m'en délivrer en quelque sorte a beaucoup contribué à la résolution que j'ai prise d'écrire mes confessions. ${ }^{36}$

Expier sa faute bien sûr, c'est donc en être déchargé. C'est pour mourir en paix, pour arriver digne au jugement dernier que Rousseau se consacre à cet exercice qu'il estime pourtant si honteux et parfois si ridicule. Chez Rousseau aussi, comme chez Augustin, la confession est un acte performatif : son énonciation publique correspond à l'adhésion immédiate au Royaume de Dieu. La publicité de l'aveu littéraire correspond à son caractère expiatoire. Et cette expiation est elle-même le signe de la conversion chrétienne et du pardon divin. L'interlocuteur de la confession n'est que secondairement la masse du lectorat potentiel : ce n'est pas le lecteur de la confession mais Dieu qui détient le pouvoir d'absoudre. Expier et être absous trouvent leur effectuation dans la confession où expier, c'est avouer publiquement devant les hommes, et être absous, avouer humblement devant Dieu. Que l'expiation ne soit que le moyen d'une fin, l'absolution, signale que l'aveu public n'est lui-même que le moyen d'une fin : l'aveu devant Dieu.

\section{Freud ou le pouvoir de la confession}

L'invention, en négatif, du sujet engage nécessairement toute philosophie du sujet sur les voies de la culpabilité, du péché et de la faute? Est-ce que le dire de soi sera nécessairement assigné à la forme de la confession? Phénoménologiquement en tout cas, le dire de la faute et la culpabilité déterminent les rapports entre l'homme et le monde, entre le Je et la collectivité. La confession comme dire de soi, comme récit en première personne adressé à Dieu et aux hommes, organise nouvellement le monde et l'ordre des pouvoirs. Augustin ne détermine peut-être pas toute philosophie du sujet comme philosophie de la responsabilité, de la culpabilité et de la conscience morale. Mais il détermine l'entrée du pouvoir, du contrôle et de la censure à l'intérieur même de l'homme. Dès lors qu'il est évident que tout homme pèche et que l'aveu du péché constitue ou permet sa rémission, l'incitation à parler de soi s'institutionnalise. Pour les Grecs, le

36. Jean-Jacques Rousseau, Les Confessions, Paris : Gallimard, Bibliothèque de la Pléiade, p. 6 
monde intérieur, la vie privée, n'avaient aucun statut et n'accédaient pas au langage. Avec Augustin, on en arrive à un stade où le dire, et donc le pouvoir, s'emparent du privé. Rien, si c'est possible, ne doit échapper à la formulation. La religion chrétienne inscrit comme devoir fondamental la tâche de faire passer tout ce qui a trait au monde privé au moulin sans fin de la parole. On avoue ses crimes, on avoue ses péchés, on avoue ses pensées et ses désirs secrets, on avoue son passé, on avoue son enfance, on avoue ses maladies et ses défauts; on s'emploie avec la plus grande exactitude à dire ce qu'il y a de plus difficile à dire; on avoue en public ou en privé, à ses parents, à son médecin, à son psychanalyste; on se fait à soi-même, dans le plaisir et dans la peine, des aveux impossibles, et dont on fait des livres.

Le pouvoir pénètre dans la vie privée; il y a désormais un contrôle moral de chacun de nos gestes, de chacune de nos pensées secrètes. II va s'agir depuis d'enserrer le moi dans une trame de discours qui tantôt s'adressent à lui, tantôt parlent de lui, tantôt imposent sur lui des connaissances canoniques et forment à partir de lui un savoir qui lui échappe et qui est d'une certaine manière toujours un contrôle, une répression. Le discours a servi à définir la norme et à caractériser toutes les déviances possibles et surtout toutes les déviances inévitables à cette norme. Avec Freud, le pouvoir pénètre encore plus avant, non seulement sur le moi conscient mais aussi sur tous les actes manqués, les lapsus, les rêves qui trahissent l'inconscient ${ }^{37}$. II ne s'agit plus seulement de dire et d'avouer

37. Il est d'ailleurs intéressant de voir à quel point les mécanismes qui ont fait de la pratique de l'aveu une forme de science selon Foucault corresponde effectivement avec la pratique psychanalytique qu'il semble implicitement viser dans sa condamnation de l'aveu en occident : Comment, demande Foucault, est-on parvenu à constituer cette immense et traditionnelle extorsion de l'aveu sexuel dans des formes scientifiques? “(...) Par une codification clinique du "faire parler" : combiner la confession et l'examen, le récit de soi-même avec le déploiement d'un ensemble de signes et de symptômes déchiffrables; l'interrogatoire, le questionnaire serré, l'hypnose avec le rappel des souvenirs, les associations libres : autant de moyens pour réinscrire la procédure d'aveu dans un champ d'observations scientifiquement acceptables. (...) Par la méthode d'interprétation : s'il faut avouer, ce n'est pas seulement parce que celui auquel on avoue aurait le pouvoir de pardonner, de consoler et de diriger. C'est que le travail de vérité à produire, si on veut scientifiquement le valider, doit passer par cette relation. Elle ne réside pas dans le seul sujet qui, en avouant, la porterait toute faite à la lumière. Elle se constitue en partie double : présente mais incomplète, aveugle à elle-même chez celui qui parle, elle ne peut s'achever que chez celui qui recueille. À lui de dire la vérité de cette vérité obscure : il faut doubler la révélation de l'aveu par le déchiffrement de ce qu'il dit. (...) Par la médicalisation et par la cure : Le vrai, s'il est dit à temps, à qui il faut, et par 
tous ses péchés, puisque ceux-ci en cachent d'autres, originaires, et qui ont été enfouis dans les profondeurs de l'inconscient et du refoulé. II ne s'agit plus seulement d'exiger des aveux en portant un soupçon systématique sur ce que l'on a naturellement tendance à taire et à garder secret, mais aussi de contester les propos conscients du patient en postulant qu'ils forment une chaîne de résistance et de souvenirs-écrans barrant l'accès au refoulé. Tout, dès lors, devient symptôme, et tout est soumis à l'acuité de l'analyste et à son jugement. Ce postulat psychanalytique fondamental de la résistance naturelle et évidente du patient à la cure contribue au pouvoir de l'analyste. L'analysé est dépossédé du sens de ses propres mots et de ses souvenirs personnels, sens qui lui est retrocédé par l'analyste. II s'agira pour l'analyste détenteur de tous les pouvoirs, de délimiter dans ce qui lui est dit "ce qui doit être cru ${ }^{38}$ ", ce qu'on «doit appeler vérité» de ce qui n'est qu' «illusion" ou "délire» du patient. ${ }^{39}$ C'est essentiellement grâce à deux procédés que le pouvoir exercé sur l'individu trouve à se développer en psychanalyse : 1) le procédé classique de l'extorsion de l'aveu et 2) le nouveau procédé de l'interprétation des aveux.

\title{
Extorsion de l'aveu
}

À la fin des Études sur l'hystérie Freud présente un de ses "petits tours de main techniques" propre à extorquer l'aveu sans avoir à torturer pour autant le patient, mais en se basant plutôt sur la manipulation :

\begin{abstract}
J'informe mon patient que je vais, dans l'instant qui suivra, exercer une pression sur son front, je l'assure que, pendant tout le temps que durera cette pression, un souvenir surgira sous la forme d'une image ou se présentera à son esprit. Mais, ajoute Freud, je lui fais obligation de me faire part de cette image ou de cette idée, quelles qu'elles puissent être. II ne doit pas les taire, même s'il pense qu'elles n'ont aucun rapport avec ce que l'on recherche, qu'il ne s'agit pas de cela ou encore qu'il les trouve désagréable à révéler. Aucune critique, aucune réserve, même pour des raisons d'affection et de mésestime! Alors, dit Freud, j'exerce pendant quelques secondes une pression sur le front du malade allongé devant moi et lui demande ensuite, d'un ton tranquille, comme si la déception était impossible : Qu'avez-vous vu ou à quoi avez-vous pensé? ${ }^{40}$
\end{abstract}

celui qui en est à la fois le détenteur et le responsable, guérit» (Foucault, op.cit., p. 87-90).

38. Sigmud Freud, L'homme Moise et la religion monothéiste, p. 234.

39. Ibid., p. 235.

40. Sigmud Freud, Études sur l'hystérie, Paris : P.U.F., p. 217-218. 
II s'agit de faire parler le patient à tout prix. Et on trouve d'ailleurs assez symptomatiquement dans le vocabulaire utilisé par Freud un emploi fréquent de la notion d'aveu. ${ }^{41}$

Dans le cas de l'homme aux rats, un petit incident est indicateur de l'incitation à dire : le malade raconte son service militaire, il raconte notamment une des manœuvres, où à la première halte, le capitaine raconte qu'il a lu la description d'un supplice particulièrement atroce qu'on pratiquait en Orient. À ce moment, le patient s'interrompt et demande à Freud de le dispenser de la description des détails. Freud refuse avec une pirouette :

Je l'assure que je n'ai moi-même aucun penchant à la cruauté, que je ne voudrais certes pas le tourmenter, mais que je ne peux le dispenser (''allemand dit Schenken) de choses dont je ne dispose pas. II pourrait tout aussi bien me demander de lui faire cadeau (Schenken toujours) de deux comètes. Vaincre les résistances est une condition du traitement à laquelle nous n'avons pas le droit de nous soustraire. 42

\section{Et cette résistance qu'il faut vaincre fait même partie des acquis}

41. J'en donne quelques exemples, tirés d'analyses de cas : "S'il est exact que l'hystérie ait sa source dans l'intimité de la vie psychique sexuelle des malades, et que les symptômes hystériques soient l'expression de leurs désirs refoulés les plus secrets, l'éclaircissement d'un cas d'hystérie doit nécessairement dévoiler cette intimité et trahir ces secrets. II est certain que les malades n'auraient jamais parlé s'ils avaient pensé à la possibilité d'une exploitation scientifique de leurs aveux..." (Cinq psychanalyses, Paris : P.U.F., p. 2) Dans une analyse de cas, Freud parle d'cobtenir de l'enfant (le petit Hans) de tels aveux" (Cinq psychanalyses, op. cit., p. 93), et il s'agira pareillement d'obtenir d'un autre cas traité par Freud, celui de "l'homme aux rats", laveu de celui-ci de ses pensées secrètes : alors qu'il vient d'exposer le supplice des rats qui signale le début de sa névrose obsessionnelle, et qu'il signale que deux idées atroces lui étaient alors venues en tête, Freud tente de savoir de quelles idées il veut parler. "L'homme aux rats" répond que son esprit a été traversé par l'idée que cela arrivait à une personne qui lui était chère, que Freud lui suggère être sa compagne. Idée à laquelle il acquiesce. Mais Freud s'interroge ensuite "car, dit-il, nous n'avons jusqu'à présent entendu parler que d'une seule idée, celle que la dame subissait le supplice aux rats. II dut alors avouer qu'une autre idée avait surgi en lui en même temps que la première, l'idée que ce supplice s'appliquait aussi à son père. Etant donné que son père était mort depuis longtemps, que cette appréhension était par conséquent encore plus absurde que l'autre, il avait essayé d'en différer encore l'aveu". (Cinq psychanalyses, op. cit., p. 207208.) Mais le vocabulaire utilisé ne peut réellement servir d'argument, il peut tout au plus corroborer d'autres constats. À savoir la première et même la seule règle du traitement : l'injonction à dire tout, et réellement tout, ce qui passe par sa tête.

42. Sigmud Freud, Cinq psychanalyses, op. cit., p. 206-207. 
théoriques de la psychanalyse. II va de soi que le patient ne dit pas tout : premièrement, le malade garde pour lui «une partie de ce qui lui est bien connu" et qu'il "devrait raconter, ceci consciemment, à dessein, pour des motifs de timidité et de pudeur" qu'il n'a pas encore surmontés (discrétion lorsqu'il s'agit d'une tierce personne). "Telle est la part de l'insincérité consciente". Deuxièmement, une partie de son savoir mnémonique, partie dont le malade dispose habituellement, fait défaut pendant le récit, sans que le malade ait l'intention de faire cette réserve : voilà la part "d'insincérité inconsciente". Troisièmement, on trouve également les "amnésies véritables", les "lacunes de la mémoire", auxquels sont sujets même des souvenirs tout récents et on trouve enfin des "erreurs de la mémoire, édifiées secondairement pour en combler les lacunes ${ }^{43}$ ".

\section{Interprétation de l'aveu}

Si la psychanalyse est forme de l'aveu, c'est qu'elle refuse la première manière de ne pas tout dire, elle refuse le secret volontaire par l'injonction à dire tout ce qui passe par la tête même les pensées les plus incohérentes ou les plus difficiles à "avouer». Si elle est aussi une pratique herméneutique de l'aveu, c'est qu'elle tend à vaincre également - c'est même tout l'objet de la cure - la deuxième forme de résistance, l'amnésie inconsciente, involontaire. Le principe d'interprétation de l'aveu repose sur la conviction que le dire du patient est toujours parcellaire et qu'il ne fait qu'affleurer à la surface de l'inconscient où séjournent les motifs réels de son discours. C'est au psychanalyste que revient la tâche de trouver ces pensées inconscientes auxquelles les propos conscients se réfèrent implicitement, mais aussi et surtout celles auxquelles puisent leur source et leur sens tous les dysfonctionnements moteurs et verbaux, les rêves, les actes symptomatiques et les résistances qui constituent le langage propre de linconscient. Prenons un exemple pour illustrer la méthode herméneutique de Freud, celui de l'homme aux rats : un jour pendant une villégiature, l'homme aux rats a lidée qu'il est trop gros et qu'il doit maigrir. II est pris alors d'une frénésie, se lève de table au dessert, se précipite en pleine chaleur d'août, sans chapeau, dans la rue, pour gravir les montagnes en courant et s'arrêter, épuisé et baigné de sueur. La solution de cette absurde compulsion, Freud la trouve dans une association ingénieuse. Son patient lui avait avoué qu'à cette époque son amie séjournait au même endroit, mais en société d'un cousin anglais qui lui faisait la cour et dont il était très

43. Sigmud Freud, Cinq psychanalyses, Paris : P.U.F., p. 9. 
jaloux. Ce cousin se nommait Richard, et tout le monde l'appelait Dick, comme c'est de coutume en Angleterre. Freud conclut : c'est ce Dick qu'il a voulu tuer (Dick, en allemand, signifie : gros.). C'est pour tuer "le gros" qu'il fallait faire un régime.

Le soupçon psychanalytique porté sur le discours du patient va si loin que Freud avoue sa perplexité lorsque celui-ci est cohérent et finalement fort "normal». "Quand surgit, pendant le traitement psychanalytique, une suite d'idées correctement fondées et impeccables, il y a pour le médecin un instant d'embarras", avoue Freud, embarras qu'il résout d'une manière caractéristique en admettant que :

(...) de telles idées, inattaquables par l'analyse, ont été utilisées par le malade pour en masquer d'autres qui voudraient se soustraire à la critique et à la conscience. Une série de reproches contre d'autres personnes laisse supposer une série de reproches de même nature dirigés contre soi-même (remords). II suffit de retourner chacun de ces reproches contre la personne même de celui qui les énonce. ${ }^{44}$

Tout propos est donc source de soupçon, mais plus encore : tout geste, tout mouvement, tout frétillement, tout gloussement. Tout est soumis à l'attention tyrannique de l'analyste. Freud remarque même qu'il a été heureusement surpris par la facilité de l'entreprise lorsqu'il s'est fixé la tâche de déterrer tout ce que les patients cachent consciemment ou non :

\begin{abstract}
Il y a dans la vie beaucoup de ...symbolisme, à côté duquel nous passons sans y prêter attention. Quand je m'imposai de ramener au jour tout ce que les hommes cachent, sans utiliser pour ce faire la contrainte qu'impose l'hypnose et en me servant simplement de ce qu'ils disent et laissent entrevoir, je croyais cette tâche plus nisilaisée qu'elle ne l'est réellement. Celui qui a des yeux pour voir et des oreilles pour entendre constate que les mortels ne peuvent cacher aucun secret. Celui dont les lèvres se taisent bavarde avec le bout des doigts; il se trahit par tous ses pores. C'est pourquoi la tâche de rendre conscientes les parties les plus dissimulées de l'âme est parfaitement réalisable. 45
\end{abstract}

44. Sigmud Freud, Cinq psychanalyses, Paris : P.U.F., p. 23.

45. Et on peut de nouveau trouver quelques exemples d'interprétation de toute cette symbolique à l'œuvre dans chacun de nos gestes, notamment celui de Dora, une jeune fille de dix-huit ans que Freud traite pour hystérie : "Le sac à main bifolié de Dora n'est autre chose que la figuration du vagin; en jouant avec ce sac, en l'ouvrant, en y introduisant le doigt, elle exprimait d'une façon assez sans-gêne, mais évidente, ce qu'elle eût voulu faire, c'est-à-dire la masturbation." (ibid., p. 56). 
L'espace de liberté se referme autour du patient dont chacun des mots et chacun des gestes se trouvent confisqués pour compléter le tableau de la névrose. II devient impossible de poser des gestes spontanés, dont la cause ne remonterait pas à quelque souvenir traumatique refoulé. II est impossible d'échapper à la causalité et donc, par définition, d'être libre. Mais de quel côté est le pouvoir? Non pas du côté de celui qui parle et impose son discours comme discours de vérité : il est contraint et non contraignant. Le pouvoir réside du côté de celui qui écoute et se tait, qui interroge et qui n'est pas censé savoir et non pas du côté de celui qui fait réponse et qui sait. Ne dit-on pas d'ailleurs que l'interlocuteur est le confesseur, et le locuteur, le confessé? D'autre part, si le pouvoir est du côté de celui qui force la confession ou qui l'écoute, celle-ci ne prend effet que dans celui à qui on l'arrache et non pas dans celui qui la suscite. La confession ne prend effet que dans la soumission, dans la reconnaissance que celui qui avoue peut prendre de sa propre faute et de ses écarts à la norme. La confession n'est activée que dans la résistance vaincue, elle est toujours le signe d'une dépossession, d'un envahissement de l'intériorité par une loi extérieure.

\section{Conclusion}

À travers cette hypothétique genèse que l'on pourrait qualifier d'historico-phénoménologique, trois caractéristiques de la confession ont été mises en relief et explicitées : la confession comme discours en première personne, comme acte performatif et comme instauration d'un pouvoir de juge et d'interprète du confesseur. $1^{\circ}$ Autobiographique, la confession est la marque de la naissance de la conscience de soi. Elle est le moteur de l'émergence de la subjectivité consciente d'elle-même à travers le conflit des volontés et de l'impossibilité devant laquelle elle se trouve de maîtriser ces pulsions internes qui la travaillent. La conscience de soi serait donc d'abord conscience de la non-maîtrise de soi. Non-maîtrise que le contexte chrétien assimilera à la faute, à l'autonomie de l'homme par rapport à Dieu et au Verbe divin et qu'Augustin assimilera plus précisément à l'abandon du Maître Intérieur. La Maîtrise de soi réside dans la possession du Maître Intérieur, la non-maîtrise, dans son oubli. La faute est toujours constituée par la désobéissance à l'autorité divine et par l'oubli de Dieu. De là, peut-être faut-il tirer la genèse des premières confessions occidentales : la conscience de la non-maîtrise rejoint celle de l'abandon fautif du Maître Intérieur et s'avoue en première personne. Et, si la faute s'avoue en 
première personne, c'est précisément qu'elle est nécessairement une faute personnelle. L'abandon et l'oubli de la Loi divine doivent forcément m'être attribués à moi et non à une quelconque démission divine. Ce n'est pas mon Maître qui m'a déserté, c'est moi qui me suis intérieurement déserté pour fuir à l'extérieur. Voilà pourquoi la confession des fautes se fait toujours à Dieu et en première personne : c'est moi qui toujours ai fauté et $c^{\prime}$ est toujours par rapport à la Loi divine que j'ai fauté. $2^{\circ}$ Mais, en tant qu'acte performatif, la confession marque elle-même la limite de l'état de faute qu'elle dénonce et avoue. Elle signale la recollection de la mémoire, la fin de l'oubli de Dieu. La confession se lie étroitement à la conversion : reconnaissant enfin sa démission chrétienne, le "je" de la faute rejoint sa patrie. Le discours même de l'aveu, de la confession qu'il tient devant Dieu et devant les hommes le rallie inaltérablement à la Cité de Dieu. Que j’aie assumé comme résolument miennes les errances de ma vie passée et que j'aie enfin pris la responsabilité de la faute qui m'incombe, me réintègre d'office au royaume de Dieu et me promet le pardon. Le "je" de la faute assumée pleinement est aussi le «je» du pardon de Dieu, l'intériorité reconquise par Dieu. Et c'est d'avoir assumé comme miennes les errances de ma vie passée que je peux réintégrer mon intériorité, mes doutes apaisés enfin et tuer en moi le conflit des volontés. C'est ici que les confessions chrétiennes d'Augustin rejoignent au plus près les confessions thérapeutiques de la psychanalyse, en consacrant la possession de soi à travers la dépossession que constitue tout aveu ${ }^{46} .3^{\circ}$ Aveu à l'Autre de ce que Je suis, la confession me dépossède moi-même. Avouant à l'autre la faute qui me constitue comme "Je", je m'en dessaisis et laisse à l'interlocuteur de ma confession le droit de l'avaliser ou non. Ai-je dit toute la vérité et seulement elle, me suis-je caché quelque chose à moi-même, telles sont les questions à travers lesquelles "Je" laisse l'autre le pénétrer. Si je deviens moi-même, c'est dans un discours en première personne bien sûr; mais ce discours ne fait jamais que suivre ou plutôt précéder les attentes du confesseur. Je ne suis je que dans un discours dont les règles sont dictées de l'extérieur. C'est un impératif moral externe qui me constitue en intimité.

Acte autobiographique, la confession me constitue comme sujet du discours et, aveu des fautes, elle constitue comme mauvaise conscience, la

46. À préciser cependant que la première personne des confessions littéraires correspond plutôt à l'auto-analyse puisque Dieu est tel que je l'ai posé en face de moi et puisque, différent en cela de l'analyste, il reste muet et me laisse seul à ma confession. 
conscience que j'ai de moi-même. Acte performatif, elle allie directement l'absolution des fautes à leur confession. Acte littéraire, elle ne pose le «je» qu'en fonction d'un «tu» qui pénètre d'emblée l'intimité du sujet et assigne au discours sa valeur de vérité.

Gaëlle Jeanmart,

FNRS, Université de Liège 